\title{
Multiuser Detection in DS-CDMA using Hybrid Evolutionary Strategy
}

\author{
Mehtaz Sharmin and Chintha Tellambura, \\ Department of Electrical and Computer Engineering \\ University of Alberta, Edmonton, AB, Canada T6G 2V4 \\ Email: \{msharmin, chintha\}@ece.ualberta.ca
}

\begin{abstract}
A hybrid Evolutionary Algorithm is presented in this paper for multiuser detection in a synchronous direct sequence code-division multiple access (DS-CDMA) system, which explores the application of Evolutionary algorithm (EA). The algorithm employs a $(1+\lambda)$ Evolutionary Strategy (ES) for the basic search, which directs the search process towards an elitist solution space. A simple 1-opt local search is applied for thorough search in the elitist region. Simulation results demonstrate the efficacy of the proposed algorithm as this approach offers near optimal bit-error rate (BER) performance with lower computational complexity compared to the maximumlikelihood (ML) detector for a large number of users.
\end{abstract}

\section{INTRODUCTION}

Direct sequence code-division multiple access (DS-CDMA) systems provide high spectrum efficiency, increased capacity, and dynamic allocation of bandwidth. In CDMA, several users access the same spectrum jointly and concurrently. Multiple access interference (MAI) is a major performance limiting factor of the conventional single user detector in a DSCDMA system. To reduce the MAI and combat the nearfar problem, Verdu introduced multiuser detection (MUD) strategy for CDMA systems which achieves the minimum probability of error [1]. He proposed a $K$-user optimal ML sequence detector for a CDMA system, which consists of a bank of $K$ single user matched filters followed by a Viterbi decoder with $2^{K-1}$ states and complexity per binary decision is $O\left(2^{K}\right)$ [2]. The ML-MUD maximizes the joint probability by evaluating the cost function over the set of all feasible user symbol sequences, resulting in a non-deterministic polynomial time hard (NP-hard) optimization problem in the number of users, $K$. However, practical implementation of such a detector is not possible for a large number of users as complexity of the optimal multiuser detector grows exponentially with the number of users.

Several suboptimum detectors have thus been proposed in the last decade where researchers have focused on developing reliable suboptimal methods with polynomial complexity. Recently researchers have considered employing artificial intelligence techniques to solve the MUD problem. The optimal ML problem is an NP-hard combinatorial optimization (CO) problem [3] which can be solved either by exact methods or by finding approximate solutions. Exact methods such as the branch and bound algorithm have been proposed to solve MUD problem in [4]. To approximately solve the MLMUD problem, heuristic methods can also be applied. A heuristic is a method that finds near-optimal solutions in a short time without being able to guarantee either feasibility or optimality [5]. In practice, much research has been carried out on heuristic methods for solving combinatorial problems [5], which give high-quality solutions and are designed to find global optima.

Heuristic methods such as Genetic Algorithm (GA), Evolutionary Programming (EP), Evolutionary Strategy (ES), Tabu search (TS), Ant Colony Optimization (ACO) have previously been employed for solving the MUD problem [6]-[12]. Neighborhood search methods like 1-opt, $k$-opt local search have been introduced to solve ML-MUD problem [13]-[15]. A GAbased detection strategy was used in [6], [8] for a multiuser receiver and it illustrated the feasibility of GA as a powerful solution for MUD problems. EP was proposed in [9] and showed that it always converges to an optimal solution with a small number of generations and has lower complexity than GA. Wang, Zhu and Kang proposed a new MUD algorithm based on $(1+\lambda)$ evolution strategy for asynchronous DSCDMA system in [10]. Simulation results of [10] showed that their algorithm provides better performance when the number of users is large, while other evolutionary algorithm based multiuser detectors may perform poorly.

Due to the incredible demand for wireless systems and increasing requirement of bandwidth efficiency, researchers have been motivated to develop effective detectors for systems with a large number of users that show better performance/complexity tradeoffs. Inspired by this challenge, a new hybrid EA is proposed in this paper by combining simple 1opt local search with $(1+\lambda)$ Evolutionary Strategy suggested in [10] to solve the ML-MUD problem, which improves the detection performance. Computer simulation reveals that the proposed method outperforms the $(1+\lambda)$ ES in [10] as 1opt local search is assisted to attain a global optimum. The computational complexity is also investigated and compared.

This paper is organized as follows. Section II describes the DS-CDMA system and the ML MUD problem. Section III gives the overview of Evolutionary Strategy and local search methods. The proposed hybrid ES for the ML detector is discussed in Section IV. In Section V, the proposed detector is compared with several existing multiuser detectors in terms of the BER performance and computational complexity. Conclusions are presented in Section VI. 


\section{DS-CDMA System Model ANd PRoblem FORMULATION}

Consider a synchronous single carrier DS-CDMA system of $K$ users sharing an additive white Gaussian noise (AWGN) multi-access channel. For simplicity, we assume that all transmitted signals are equally probable and also assume that $K$ users simultaneously transmit BPSK signals through a slowly fading channel. Each user is assigned a pseudorandom signature sequence $s_{k}(t)$ of duration $T_{s}$, where $T_{s}$ is the symbol interval. A signature sequence can be expressed as

$$
s_{k}(t)=\sum_{j=0}^{L-1} c_{k}(j) p\left(t-j T_{c}\right), \quad\left(0 \leq t \leq T_{s}\right)
$$

where $\left\{c_{k}(j),(0 \leq j \leq L-1)\right\}$ is a code sequence consisting of $L$ chips that takes value $\{ \pm 1\}$, and $p(t)$ is the pulse with duration $T_{c}$, where $T_{c}$ is the chip interval. The spreading gain is $L=T_{s} / T_{c}$. The information sequence of $k$-th user is denoted by $\left\{b_{k}(m)\right\}$, where the value of each information symbol is $\{ \pm 1\}$. Let us consider a block of $M$ transmitted symbols. The received signal for synchronous transmission may be expressed as

$$
r(t)=\sum_{k=1}^{K} \sqrt{\varepsilon_{k}} \sum_{m=1}^{M} b_{k}(m) s_{k}(t)+n(t)
$$

where $\varepsilon_{k}$ represents the energy of the $k$-th user signal, $n(t)$ is noise with mean zero and with power spectral density $N_{0} / 2$ [16].

The received signal $r(t)$ is demodulated by a bank of matched filters. Considering received signal in one signal interval $0 \leq t \leq T_{s}$, the sampled output of the matched filter of the $k$-th user is given by

$$
y_{k}=\sqrt{\varepsilon_{k}} b_{k}(1)+\sum_{k \neq l} \sqrt{\varepsilon_{l}} b_{l}(1) \rho_{l k}(0)+n_{k}
$$

where the cross-correlation term $\rho_{l k}(0)=\int_{0}^{T_{s}} s_{l}(t) s_{k}(t) d t$ and the noise term $n_{k}=\int_{0}^{T_{s}} n(t) s_{k}(t) d t$. Arranging in vector format for all $K$ users, the matched filter (MF) outputs can be expressed as

$$
\mathbf{y}=\mathbf{R} \mathbf{\Sigma} \mathbf{b}+\mathbf{n}
$$

where MF outputs are $\mathbf{y}=\left[y_{1}, y_{2}, \ldots . y_{K}\right]^{T}, \mathbf{R}$ is symmetric positive definite correlation matrix with elements $\rho_{l k}(0), \boldsymbol{\Sigma}$ is a diagonal matrix i.e. $\boldsymbol{\Sigma}=\operatorname{diag}\left(\sqrt{\varepsilon_{1}}, \sqrt{\varepsilon_{2}}, \ldots \ldots \ldots, \sqrt{\varepsilon_{K}}\right)$, the information vector $\mathbf{b}=\left[b_{1}, b_{2}, \ldots . . b_{K}\right]^{T}$ and $\mathbf{n}=$ $\left[n_{1}, n_{2}, \ldots \ldots, n_{K}\right]^{T}$ is a noise vector of zero mean Gaussian random variables whose covariance matrix is $\mathrm{E}\left[\mathbf{n n}^{T}\right]=$ $\frac{N_{0}}{2} \mathbf{R}=\sigma^{2} \mathbf{R}$.

The optimum ML receiver decides on $\mathbf{b}$ given an observed vector $\mathbf{y}$ using the Maximum Likelihood criterion by maximizing the conditional probability:

$$
\hat{\mathbf{b}}=\underset{\mathbf{b} \in\{-1,+1\}^{K}}{\operatorname{argmax}} p(\mathbf{y} \mid \mathbf{b}) .
$$

So, $\hat{\mathbf{b}}$ is the maximum-a-posterori (MAP) estimate for the transmitted vector $\mathbf{b}$ given the received vector $\mathbf{y}$. When all signal vectors $\mathbf{b} \in\{-1,+1\}^{K}$ are equally probable, MAP estimation is equivalent to maximum-likelihood estimation (MLE) and the following binary constrained optimization problem arises:

$$
\hat{\mathbf{b}}_{\text {opt }}=\underset{\mathbf{b} \in\{-1,+1\}^{K}}{\operatorname{argmin}} \mathbf{b}^{T} \boldsymbol{\Sigma}^{T} \mathbf{R} \boldsymbol{\Sigma} \mathbf{b}-2 \mathbf{y}^{T} \boldsymbol{\Sigma} \mathbf{b} .
$$

Since there are $2^{K}$ information sequences of the $K$ users, search is required over all $2^{K}$ possible combinations. Thus, computational complexity of the optimum detector grows exponentially with the number of users, $K$.

\section{Overview of ES AND Local SEARCh}

For NP-hard CO problems, complete methods may have exponential time complexity in the worst-case. In the last 20 years, metaheuristics has emerged for such problems. The main idea of metaheuristic is to combine basic heuristic methods with higher level frameworks aimed at efficiently and effectively exploring a search space using the concepts derived from artificial intelligence, biological, mathematical, neural and physical sciences [17]. Such algorithms comprise Evolutionary Algorithms (EAs) including EP, ES, and GA. EAs are population-based metaheuristic optimization techniques that are used to solve a large class of problems. They originate from the inspiration of the natural process of biological evolution, the way of species evolving and adapting to their environment. Although convergence of EAs are guaranteed to a global optimum in a weak probabilistic sense, they have successfully been used to solve various complex combinatorial optimization problems.

The main attractive features of EAs are the simplicity of the algorithms as they are relatively cheap and quick to implement, and they can cope well with noisy, inaccurate data in general [18]. To identify a highly effective search space, it is necessary to include some form of domain knowledge into EAs [19]. There are several ways to achieve this. A simple and promising way is to combine local search with EAs, which increases the efficiency of EA as problem attributes can be exploited in local search to speed up the neighborhood search process. Such a hybridized algorithm is known as a memetic algorithm. EAs maintain a population of structures that evolve using operators such as selection, recombination and mutation. Each individual in the population is evaluated using a fitness function and then high fitness individuals are selected. Recombination and mutation perturb those individuals for exploration of the search space. The search method terminates through a predefined criterion.

Local search method [20] takes a reasonable solution as input and then searches for better solutions from neighborhood of the current solution by stepwise transition. The search process stops when no better solution is found in the neighborhood. The neighborhood that contains all solutions within unit Hamming distance to the current solution is known as 1-opt neighborhood i.e., 1-opt neighborhood of the current solution $\mathbf{b}$ is defined as

$$
N_{1-o p t}(\mathbf{b})=\left\{\tilde{\mathbf{b}} \in B \mid w_{H}(\mathbf{b}, \tilde{\mathbf{b}})=1\right\}
$$


where $w_{H}$ denotes the Hamming distance metric and $B=$ $\{-1,+1\}^{K}$.

\section{Proposed hybrid ES Algorithm For MUD}

ES is a probabilistic optimization technique which has several notations like $(1+1) \mathrm{ES},(1+\lambda) \mathrm{ES},(\mu+\lambda),(\mu, \lambda)$ [21]. ES emphasizes the behavioral change at the level of individuals. The proposed method combines the simplest one of the ES notations, $(1+\lambda)$ ES as in [10] with 1-opt local search. $(1+\lambda)$ ES uses a parent population size of 1 and creates $\lambda$ individuals/offsprings in each generation by replication and mutuation. Recombination and other evolutionary operators are not used in this algorithm which makes ES simpler than other EAs. The basic $(1+\lambda)$ ES can be found in [22]. It has two main steps:

1) Mutuation is done in the current solution to produce offsprings;

2) The best solution is selected from offsprings for next generation using fitness function;

For the minimization problem, in selection step, the parent is replaced by an offspring with minimum offspring fitness if and only if the minimum offspring fitness is less than or equal to the parent's fitness. The process is repeated to get better and better solutions.

When hybrid $(1+\lambda)$ ES is applied to MUD problem, the following fitness/objective function is used to evaluate offsprings:

$$
f(\mathbf{b})=\mathbf{b}^{T} \boldsymbol{\Sigma}^{T} \mathbf{R} \boldsymbol{\Sigma} \mathbf{b}-2 \mathbf{y}^{T} \boldsymbol{\Sigma} \mathbf{b} .
$$

Considering $K$-user synchronous DS-CDMA system, the hybrid ES algorithm for multiuser detector can be described as follows:

- The output of the single-user mathched filter is taken as initial solution which is the parent: $\mathbf{b}_{p}=\mathbf{b}_{\text {initial }}=$ $\hat{\mathbf{b}}_{S U M F}=\operatorname{sign}(\mathbf{y})$.

- The population size of offspring is set as: $\lambda=\lfloor K \ln K\rfloor$, where $\lfloor K \ln K\rfloor$ represents the maximum integer.

- For iteration $m=1,2, \ldots \ldots, N_{g} ; N_{g}$ is the number of generation,

1) For each $l=1,2, \ldots ., \lambda, \mathbf{b}_{l} \in\{-1,+1\}^{K}$ is created by copying $\mathbf{b}_{p}$ and independently flipping each bit with mutuation probability $P_{m}$.

2) For each individual of the $\lambda$ population, 1-opt local search is performed representing all individuals in the population as local minima. For each $\mathbf{b}_{l}, l=$ $1,2, \ldots \ldots, \lambda$, the best neighbor $\mathbf{b}_{l, b e s t}$ in the 1 -opt neighborhood $N\left(\hat{\mathbf{b}}_{l}\right)$ is searched by evaluating the objective function. If $f\left(\dot{\mathbf{b}}_{l, \text { best }}\right)<f\left(\dot{\mathbf{b}}_{l}\right)$, then $\mathbf{b}_{l} \leftarrow$ $\dot{\mathbf{b}}_{l, \text { best }}$ else $\mathbf{b}_{l} \leftarrow \mathbf{b}_{l}$.

3) Each locally minimized indiviual of the current population is then evaluated using fitness function and the individual with minimum fitness value is determined as $\mathbf{b}_{l, \min }$.

4) If $f\left(\mathbf{b}_{l, \min }\right) \leq f\left(\mathbf{b}_{p}\right)$, then $\mathbf{b}_{p} \leftarrow \dot{\mathbf{b}}_{l, \text { min }}$ else $\mathbf{b}_{p} \leftarrow$ $\mathbf{b}_{p}$.
- The whole search process is terminated after $N_{g}$ generations giving $\mathbf{b}_{\mathbf{p}}$ as the best solution.

Janson, Jong and Wegner analyzed the effect of offspring population size $\lambda$ in [22]. The influence of this parameter on the search performance of ES is very high as computation cost of fitness evaluation depends largely on this. The number of fitness function evaluations in different instances for MUD is also investigated in [10]. From those analysis, it is found that, computation cost is the least if $\lambda$ is approximately $K \ln K$ providing better convergence rate. Here, mutation is the only operator that explores the search space. Although several papers have suggested that mutation probability for ES algorithm should be $1 / n$, where $n$ is the problem size, mutation probability $P_{m}$ is chosen here as in [10] where it is shown that setting $P_{m}=0.2$ makes the ES algorithm escape from local optima efficiently.

\section{Simulation Results}

The BER performance and computational complexity of the hybrid ES based multuser detector are evaluated in this section through simulation. Synchronous DS-CDMA system with perfect power control using BPSK transmission over the AWGN channel is considered. All users are assumed to have equal average transmission signal energy. Randomly generated binary spreading sequences of length 31 are used.

The first simulation considers a 15-user system. The BER versus signal-to-noise ratio (SNR) of the proposed hybrid ES based detector is plotted in Fig. 1. BER performance of the conventional detector (CD), $(1+\lambda)$ ES based detector [10], 1opt local search based detector [14], and the optimal detector [2] are also plotted in the same figure for comparison. The optimal ML detector is implemented by exhaustive search. The search process for proposed and $(1+\lambda)$ ES based detector terminates after the predefined number of generation, $N_{g}$. For simulation, $N_{g}=K$, the number of users, is used. The BER of the proposed hybrid ES based detector is very close to that of the optimal detector and also outperform the CD, $(1+\lambda) \mathrm{ES}$ and 1-opt local search based detectors. Fig. 2 shows the same comparison of the BER performance for a 20-user system.

The hybrid ES based multiuser detector evaluates the objective function (8) $K(\lambda(K+1)+1)$ times, which is in polynomial time complexity in terms of the number of users, $K$. Fig. 3 shows that the proposed detector is able to reduce the complexity of the optimum ML detector. For example, the complexity is reduced by a factor of 42 , when the number of users is 20 . The complexity reduction factor is $2^{K} /(K(\lambda(K+$ 1) +1$)$ ).

\section{CONCLUSIONS}

In this paper, a hybrid Evolutionary Strategy employing 1opt local search in $(1+\lambda)$ ES is proposed for solving the ML-MUD problem. Simulation results show the effectiveness of the proposed method as this offers the near-optimal BER performance for a large number of users. This hybrid ES based detector is able to reduce the detection complexity in terms of function evaluation significantly compare to the optimum ML 


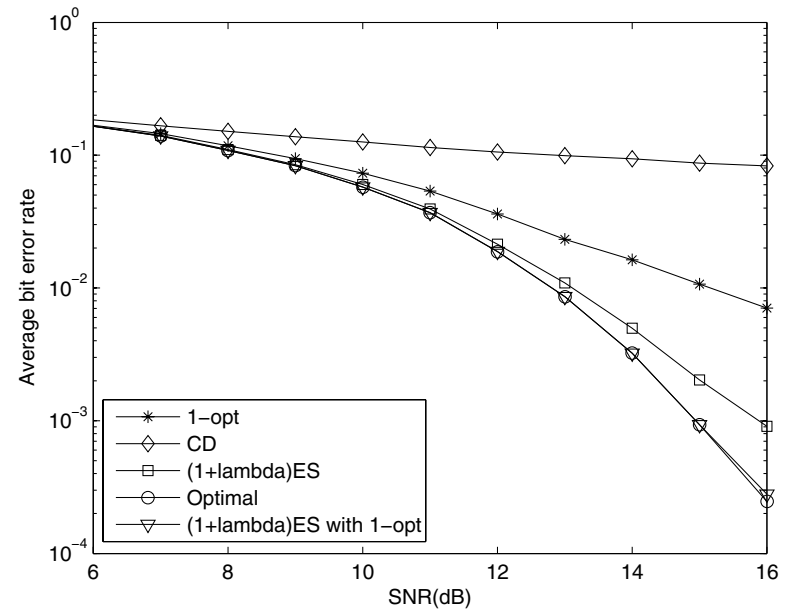

Fig. 1. Average BER of 1-opt local search, CD, $(1+\lambda)$ ES, Optimal, $(1+\lambda)$ ES with 1-opt local search for $K=15,10^{4}$ Monte Carlo runs.

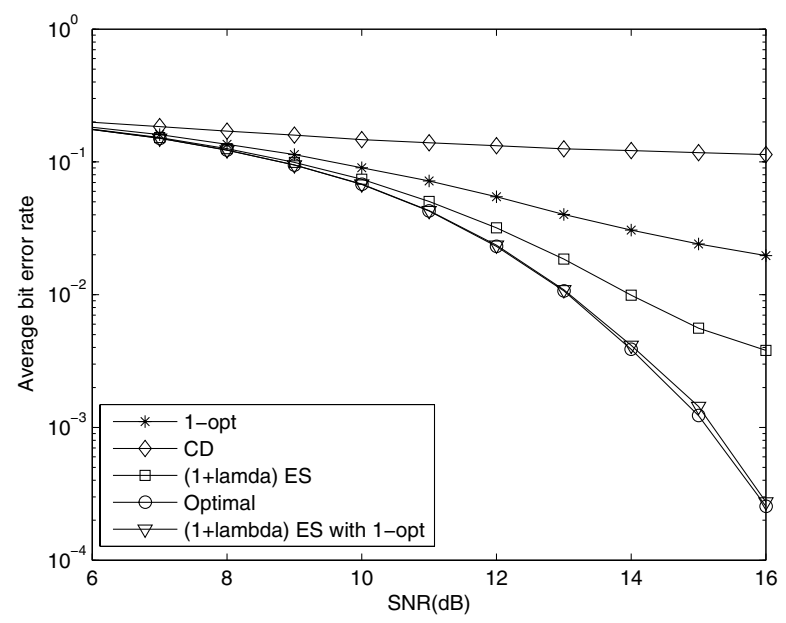

Fig. 2. Average BER of 1-opt local search, CD, $(1+\lambda)$ ES, Optimal, $(1+\lambda)$ ES with 1-opt local search for $K=20,10^{4}$ Monte Carlo runs.

detector when the number of users is higher than 20. This gain increases with the number of users.

\section{REFERENCES}

[1] S. Verdu, Multiuser Detection. Cambridge: Cambridge Univ. Press, 1998.

[2] —_ "EnglishMinimum probability of error for asynchronous gaussian multiple-access channels," EnglishIEEE Trans. Inform. Theory, vol. 32, pp. 85 - 96, Jan. 1986.

[3] S. Verdú, "Computational complexity of optimum multiuser detection," Algorithmica, vol. 4, no. 3, pp. 303-312, 1989.

[4] J. Luo, K. Pattipati, P. Willett, and G. Levchuk, "Fast optimal and suboptimal any-time algorithms for CDMA multiuser detection based on branch and bound," IEEE Trans. Commun., vol. 52, no. 4, pp. 632642, Apr. 2004.

[5] C. R. Reeves, Ed., Modern heuristic techniques for combinatorial problems. New York: Halsted Press [John Wiley \& Sons Inc.], 1993.

[6] S. Abedi and R. Tafazolli, "Genetic multiuser receiver for code division multiple access communications," Electronics Letters, vol. 36, no. 23, pp. 1957-1958, Nov. 2000.

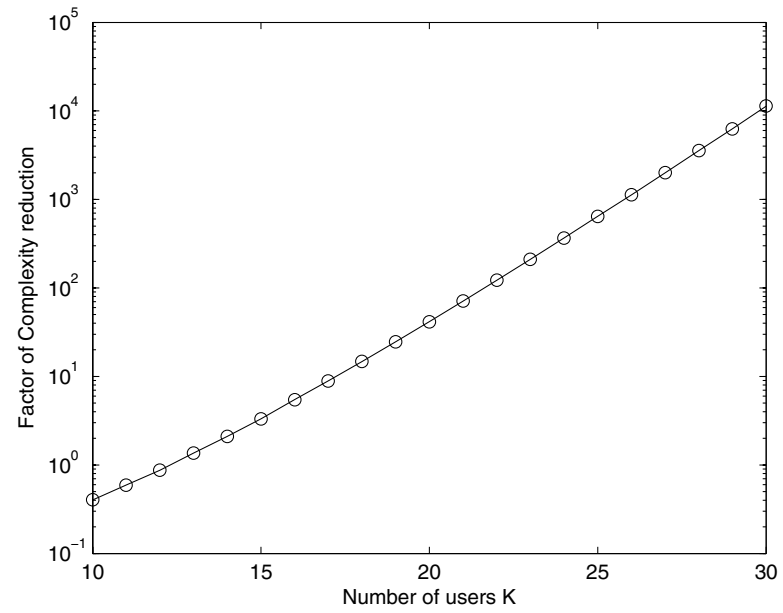

Fig. 3. The complexity reduction of the proposed algorithm in comparison to the optimal ML detector.

[7] P. H. Tan and L. Rasmussen, "Multiuser detection in CDMA - a comparison of relaxations, exact, and heuristic search methods," IEEE Transactions on Wireless Communications, vol. 3, no. 5, pp. 1802-1809, Sept. 2004.

[8] C. Ergun and K. Hacioglu, "Multiuser detection using a genetic algorithm in CDMA communications systems," IEEE Transactions on Communications, vol. 48, no. 8, pp. 1374-1383, Aug. 2000.

[9] H. Lim, M. Rao, A. Tan, and H. Chuah, "Multiuser detection for DSCDMA systems using evolutionary programming," IEEE Commun. Lett., vol. 7, no. 3, pp. 101-103, Mar. 2003.

[10] S. Wang, Q. Zhu, and L. Kang, " $1+/$ spl lambda/) evolution strategy method for asynchronous DS-CDMA multiuser detection," IEEE Commun. Lett., vol. 10, no. 6, pp. 423-425, June 2006.

[11] S. Hijazi, A. Best, B. Natarajan, and S. Das, "Ant-colony based optimal MC-CDMA multiuser detector," in Wireless And Mobile Computing, Networking And Communications, 2005. (WiMob'2005), IEEE International Conference on, vol. 1, Aug. 22-24, 2005, pp. 128-132.

[12] L. Hongwei, W. Xinhui, and L. Sanyang, "The application of nonlinear programming for multiuser detection in CDMA," IEEE Transactions on Wireless Communications, vol. 3, no. 1, pp. 8-11, Jan. 2004.

[13] G. He, A. Kot, and T. Qi, "Decorrelator-based neighbour-searching multiuser detector in CDMAsystems," Electronics Letters, vol. 32, no. 25, pp. 2307-2308, Dec. 5, 1996.

[14] J. Hu and R. Blum, "A gradient guided search algorithm for multiuser detection," IEEE Commun. Lett., vol. 4, no. 11, pp. 340-342, Nov. 2000.

[15] H. S. Lim and B. Venkatesh, "An efficient local search heuristics for asynchronous multiuser detection," IEEE Commun. Lett., vol. 7, no. 7, pp. 299-301, July 2003.

[16] J. G. Proakis, Digital Communications, 3rd ed. New York: McGraw Hill, 1995.

[17] I. H. Osman and G. Laporte, "Metaheuristics: A bibliography," Annals of Operations Research, vol. 63, pp. 513-623, 1996.

[18] G. R. Raidl, "Evolutionary computation: An overview and recent trends," OGAI Journal (Oesterreichische Gesellschaft fuer Artificial Intelligence), vol. 24, no. 2, pp. 2-7, 2005.

[19] T. Back, U. Hammel, and H.-P. Schwefel, "Evolutionary computation: comments on the history and current state," IEEE Trans. Evol. Comput., vol. 1, no. 1, pp. 3-17, Apr. 1997.

[20] V. J. Rayward-Smith, I. H. Osman, C. R. Reeves, and G. D. Smith, Eds., Modern heuristic search methods. Chichester: John Wiley \& Sons Ltd., 1996.

[21] H. Beyer and H. Schwefel, "Evolution strategies - a comprehensive introduction," Natural Computing, vol. 1, pp. 3 - 52, Mar. 2002.

[22] T. Jansen, K. A. D. Jong, and I. A. Wegener, "On the choice of the offspring population size in evolutionary algorithms," Evol. Comput., vol. 13, no. 4, pp. 413-440, 2005. 